\title{
Syphilis Self-testing: A Nationwide Pragmatic Study Among Men Who Have Sex With Men in China
}

\author{
Cheng Wang, ${ }^{1,2,3, a}$ Weibin Cheng, ${ }^{1,2, a,}$ Changchang Li, ${ }^{1,2}$ Weiming Tang, ${ }^{1,2,3}$ Jason J. Ong, ${ }^{4,5}$ M. Kumi Smith, ${ }^{6}$ Hongyun Fu, ${ }^{7}$ Michael Marks, ${ }^{4}$ Juan Nie, ${ }^{1,2}$ \\ Heping Zheng, ${ }^{1,2,3}$ Joseph D. Tucker, ${ }^{3,4,8, b}$ and Bin Yang ${ }^{1,2, b}$ \\ ${ }^{1}$ Dermatology Hospital of Southern Medical University, ${ }^{2}$ Guangdong Center for Skin Diseases and Sexually Transmitted Infection Control, and ${ }^{3}$ University of North Carolina Project-China, \\ Guangzhou, China; ${ }^{4}$ Faculty of Infectious and Tropical Diseases, London School of Hygiene and Tropical Medicine, United Kingdom; ${ }^{5}$ Central Clinical School, Monash University, Melbourne, \\ Australia; ${ }^{6}$ Division of Epidemiology and Community Health, University of Minnesota Twin Cities, Minneapolis; ${ }^{7}$ Division of Community Health and Research, Eastern Virginia Medical School, \\ Norfolk; and ${ }^{8}$ Institute for Global Health and Infectious Diseases, School of Medicine, University of North Carolina at Chapel Hill
}

Background. Syphilis self-testing may help expand syphilis testing among men who have sex with men (MSM). China has rapidly scaled up human immunodeficiency virus (HIV) self-testing, creating an opportunity for integrated syphilis self-testing. However, there is a limited literature on implementing syphilis self-testing.

Methods. A cross-sectional online survey was conducted among Chinese MSM in 2018. Participants completed a survey instrument including sociodemographic characteristics, sexual behaviors, syphilis self-testing, and HIV self-testing history. Multivariable logistic regression was conducted to identify correlates of syphilis self-testing. We also recorded potential harms associated with syphilis self-testing.

Results. Six hundred ninety-nine MSM from 89 cities in 21 provinces in China completed the study. A total of 361/699 (51.7\%) men tested for syphilis, of whom 174/699 (24.9\%) men used syphilis self-testing. Among 174 who had self-tested, 90 (51.7\%) reported that the self-test was their first syphilis test and 161 (92.5\%) reported that they undertook syphilis self-testing together with HIV self-testing. After adjusting for covariates, syphilis self-testing was correlated with disclosure of sexual orientation to family or friends (adjusted odds ratio [aOR], 1.90; 95\% confidence interval [CI], 1.32-2.73), reporting 2-5 male sexual partners (aOR, 1.81; 95\% CI, 1.04-3.16), HIV self-testing (aOR, 39.90; 95\% CI, 17.00-93.61), and never tested for syphilis in the hospital (aOR, 2.96; 95\% CI, 1.86-4.72). Self-reported harms associated with syphilis self-testing were minimal.

Conclusions. Scaling up syphilis self-testing could complement facility-based testing in China among MSM. Self-testing may increase first-time testing and has limited harms. Our findings suggest that syphilis self-testing could be integrated into HIV selftesting services.

Keywords. syphilis; self-testing; men who have sex with men; MSM.

Syphilis remains an urgent public health priority worldwide. In 2012, over 17 million syphilis cases were reported among individuals aged 15-49 years worldwide [1]. Men who have sex with men (MSM) are disproportionately affected by syphilis [2-5]. Expanding syphilis testing is a cornerstone of syphilis control $[6,7]$. However, syphilis testing uptake remains low among MSM in low- and middle-income countries (LMICs). Studies suggest that only $30 \%$ of Chinese MSM has ever received a syphilis test $[8,9]$.

Received 20 March 2019; editorial decision 26 June 2019; accepted 28 June 2019; published online July 1, 2019

${ }^{\mathrm{a}}$ C. W. and W. C. contributed equally to this work.

bJ. D. T. and B. Y. contributed equally to this work.

Correspondence: J. D. Tucker, Institute of Global Health and Infectious Diseases, University of North Carolina at Chapel Hill, Number 2 Lujing Rd, Guangzhou, China, 510095 (jdtucker@ med.unc.edu).

\section{Clinical Infectious Diseases ${ }^{\circledR} \quad$ 2020;70(10):2178-86}

(C) The Author(s) 2019. Published by Oxford University Press for the Infectious Diseases Society of America. All rights reserved. For permissions, e-mail: journals.permissions@oup.com. DOI: $10.1093 /$ cid/ciz603
Barriers preventing MSM from accessing to syphilis testing include inconvenient testing systems [10], lack of privacy [11], and stigma associated with syphilis testing [12]. Many clinics, including sexually transmitted disease clinics, are not able to perform nontreponemal syphilis tests in China [13]. Clinics that do provide syphilis testing are not financially reimbursed when compared with reimbursements related to human immunodeficiency virus (HIV) testing [14].

Syphilis self-testing may help improve test uptake among key populations. Self-testing is the process whereby a person collects a specimen, performs the test, and interprets the result themselves [9]. HIV self-testing programs have already introduced the concept of self-testing to MSM in many countries, creating an infrastructure to distribute and measure uptake of self-testing $[15,16]$. A total of 59 countries globally have policies supporting HIV self-testing [17]. Sensitive and specific syphilis self-test kits and dual HIV/syphilis self-test kits are available in China and can be used to screen for syphilis [18], similar to the treponemal serological assay commonly used in clinics [19]. Self-testing allows users to choose the location, 
time, and setting of testing, providing an opportunity for decentralized testing $[18,20]$ and alternative service delivery models $[21,22]$. Self-testing may also be associated with decreased testassociated stigma [23]. China has scaled up HIV self-testing among MSM $[18,24]$. A large number of MSM in China regularly use HIV self-testing, creating an opportunity to integrate syphilis self-testing. However, there is a limited literature on syphilis self-testing $[18,25]$. The purpose of this cross-sectional nationwide survey was to examine syphilis self-testing experience and its determinants among MSM in China.

\section{METHODS}

\section{Study Design and Participants}

An online cross-sectional survey was conducted between 14 and 28 July 2018. Links to the online survey were disseminated to potential participants by local health departments and community-based organizations through Weibo (a microblogging platform) and WeChat (a messaging app). Participants entered the survey by clicking a link, which directed them to a survey website hosted by WenJuanXing (Changsha Haoxing Information Technology Co, Ltd, China).

All participants who clicked the link for the survey were screened for eligibility. Inclusion criteria included the following: born biologically as a male, aged 16 or over, and engaged in anal or oral sex with a man at least once during their lifetime.

To minimize the risk of participants taking the survey multiple times, we utilized a mobile phone code verification process in the survey. The survey was restricted to 1 phone number and a single device. Each phone number was able to receive a verification code once and each device could only access the survey once.

\section{Syphilis Self-testing Process in China}

Syphilis self-testing kits can be accessed on e-commence platforms or through existing HIV self-testing programs in China [18]. We searched the available syphilis self-testing kits sold on the 2 largest e-commerce platforms in China (Taobao and Jingdong). There were 10 brands of syphilis self-testing kits available online. All of the kits are blood-based rapid testing kits using a colloidal gold method, which detect treponemalspecific antibodies and cannot distinguish between current and past infection. All of the kits were certified by the China Food and Drug Administration. The sensitivity and specificity are excellent (Supplementary Table S1). Several HIV/syphilis selftesting programs are supported by the Chinese government. The cost per test kit ranges from US $\$ 2.5$ to $\$ 15$. The procedure for blood-based syphilis self-testing is similar to the procedure for blood-based HIV self-testing.

\section{Measures}

\section{Sociodemographic and Behavioral Variables}

Sociodemographic information included: age, marital status, education, income, sexual orientation, and disclosure of sexuality orientation to healthcare providers or family or friends. Behavioral variables included number of male sexual partners and female sexual partner in the past 3 months, condom used in the past 3 months, illicit substance use, group sex, commercial sex with men, and HIV and syphilis testing history.

\section{Syphilis Self-testing History}

Syphilis self-testing history included the following: location where the kit was obtained, type of test kit used (single or in combination with HIV), whether the individual performed selftesting alone or together with a sexual partner (including both regular and casual partner), syphilis self-test results, posttest healthcare-seeking behaviors, and subsequent changes in syphilis testing frequency after initial use of a self-test kit.

Experiences of being pressured to take a syphilis self-test were also assessed, a known issue in the use of HIV self-test kits $[26,27]$. Pressured testing was defined as being forced to take a syphilis test against one's will through physical, verbal, or psychological pressure. Categories of pressure were defined as physical violence (eg, pushing, slapping, punching, kicks), threats of violence, verbal abuse (eg, being shouted at), psychological pressure (eg, being neglected, being discriminated), excessive control of activities (eg, not being allowed to leave the house), withholding of household resources, and threats to end a relationship [27].

For participants who reported past experience of syphilis self-testing, we requested 1 of the following 3 items to confirm their self-testing: a photograph of the receipt of purchase for a self-test kit, screenshot of the transaction record showing the purchase of a self-test kit, or a photograph of their used syphilis self-test kit.

The questionnaire is provided in Supplementary Table S2.

\section{Statistical Analysis}

We report descriptive statistics for the distribution of the sample regarding background characteristics, substance use, sexual behaviors, and HIV and syphilis testing.

Univariable and multivariable logistic regression was conducted to explore sociodemographic and behavioral variables associated with syphilis self-testing. In the multivariable model we adjusted for age, legal marital status, educational attainment, annual income, and sexual orientation. We performed a subanalysis to explore correlates of syphilis self-testing restricted to participants who had ever used HIV self-testing. Statistical significance was defined as $P<.05$. Analyses were performed using IBM SPSS Statistics (version 22).

\section{RESULTS}

Overall, the survey link was clicked 1814 times. Of these, 1036 withdrew from the survey prior to reading the consent form and 5 were excluded for not signing the consent form. Among the remaining 773 men, 59 did not meet eligibility requirements 
(13 were female, 2 were less than 16 years old, and 44 did not engage in anal or oral sex with a man during their lifetime), and 15 duplicates were excluded. A total of 699 men completed the online survey. These individuals were in 103 cities of 29 provinces (Supplementary Table S3). Among them, 361 (51.7\%) had ever tested for syphilis, of whom 174 (48.2\%) had ever used syphilis self-testing. A total of 540 (77.3\%) had ever tested for HIV, of whom 406 (75.2\%) had ever used HIV self-testing. (Figure 1)

The majority of participants were between 16 and 35 years old $(90.7 \%)$, were never married $(84.7 \%)$, self-identified as gay (70.0\%), had a college degree or higher (78\%), and had an annual income less than US $\$ 9000$ (63\%). The sociodemographic characteristics of respondents who ever used syphilis selftesting were comparable to men who never used syphilis selftesting (Table 1).

Of participants who had ever tested for syphilis, $12.2 \%$ $(44 / 361)$ reported that their most recent test result was positive and $80.3 \%$ (290/361) reported a negative result. Among those reporting ever using syphilis self-testing, 37.4\% (65/174) of participants uploaded valid proof materials. Sociodemographic characteristics were similar between individuals who had uploaded proof materials and those did not, except for legal marital status (Supplementary Table S4).

\section{Syphilis Self-testing Experience}

Among the 174 individuals who had self-tested for syphilis, more than half reported that the self-test was their first-ever syphilis test $(51.7 \%, 90 / 174)$ and self-tested together with their regular partner $(52.9 \%, 92 / 174)$. Most syphilis self-testing $(92.5 \%, 161 / 174)$ was undertaken in conjunction with HIV selftesting, and approximately half $(53.1 \%, 85 / 161)$ reported that they used separate self-test kits for HIV and syphilis in their most recent self-testing. The most common place to obtain a syphilis self-test kit was from a community-based organization $(67.2 \%, 117 / 174)$, followed by purchase from an online vendor (33.3\%, 58/174). Twenty-two individuals (12.6\%, 22/174)

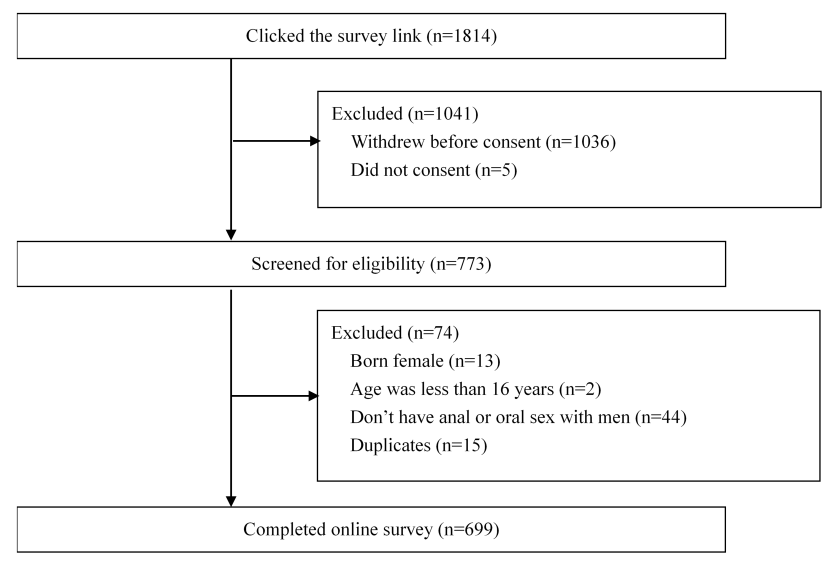

Figure 1. Flowchart of the study population. reported a reactive result in their most recent syphilis self-test. Twenty-five men $(83.3 \%, 25 / 30)$ sought care following syphilis self-testing, including 3 who reported an indeterminate result. The majority $(72 \%, 18 / 25)$ sought care within 1 month of selftesting, and three-quarters $(72 \%, 18 / 25)$ sought care either in a general hospital or at a specialty sexually transmitted infection (STI) clinic. A minority of participants $(28.2 \%, 49 / 174)$ reported increasing their syphilis test frequency after first using syphilis self-testing (Table 2).

Among individuals who had self-tested, 30 men (17.2\%, $30 / 174$ ) reported ever experiencing pressure to undertake syphilis self-testing, 25 men $(69.4 \%, 25 / 36)$ reported that their most recent experience of being pressured was from a regular male sexual partner. The most common forms of pressure were verbal abuse $(33.3 \%, 10 / 30)$ and a threat to end a relationship (33.3\%, 10/30). Physical violence was reported by 6 men $(3.4 \%)$ (Table 2).

\section{Factors Correlated With Syphilis Self-testing}

In the multivariable model adjusted for age, legal marital status, educational attainment, annual income, and sexual orientation, 5 factors were positively associated with uptake of syphilis self-testing: disclosure of sexual orientation to family or friends (aOR, 1.90; 95\% CI, 1.32-2.73), reporting 2 to 5 male sexual partners in the last 3 months (aOR, 1.81; 95\% CI, 1.04-3.16), past HIV testing (aOR, 20.07; 95\% CI, 7.20-55.97), past HIV self-testing (aOR, 39.90; 95\% CI, 17.00-93.61), and never tested for syphilis in the hospital (aOR, 2.96; 95\% CI:,1.86-4.72). In the subgroup analysis of individuals who had ever used HIV self-testing, 4 factors were positively associated with uptake of syphilis self-testing: having a female sexual partner in the past 3 months (aOR:,2.11,;95\% CI, 1.02-4.34), having ever paid for commercial sex (aOR, 2.18,;95\% CI, 1.22-3.92), having ever sold sex to men (aOR, 3.14,;95\% CI, 1.65-5.96), and never having tested for syphilis in the hospital (aOR:,3.30; 95\% CI, 1.91-5.71) (Table 3).

\section{Difficulties and Reasons for Performing Syphilis Self-testing}

Overall, 39.1\% (68/174) of self-testers reported some forms of difficulty in performing self-testing. The most commonly reported difficulty was pricking the finger to obtain a blood sample $(31.5 \%, 53 / 68)$. The most common reasons for not using syphilis self-testing was lack of knowledge of where to obtain a self-test kit $(21.2 \%, 199 / 525)$ or lack of knowledge of the availability of syphilis self-testing $(20.6 \%, 193 / 525)$. The most common reason for performing syphilis self-testing was men reporting that they wished to know their infection status $(41.6 \%, 96 / 174)$ (Supplementary Table S5).

\section{DISCUSSION}

Syphilis self-testing may reach people who do not seek testing in a facility-based setting. Our data suggest that potential harms 
Table 1. Social Demographic and Sexual Behavioral Characteristics of Participants Among Men Who Have Sex With Men: A Nationwide Cross-sectional Survey in China, 2018

\begin{tabular}{|c|c|c|c|c|}
\hline Characteristics & Total $(\mathrm{N}=699)$ & Self-tester $(n=174)$ & Facility-tester $(n=191)$ & Nontester $(n=334)$ \\
\hline \multicolumn{5}{|l|}{ Age } \\
\hline $16 \sim 25 y$ & $318(45.5)$ & $72(41.4)$ & $72(37.7)$ & $174(52.1)$ \\
\hline $26 \sim 35$ y & $316(45.2)$ & 85 (48.9) & $91(47.6)$ & $140(41.9)$ \\
\hline $36 \sim 45 y$ & $47(6.7)$ & $13(7.5)$ & $21(11.0)$ & $13(3.9)$ \\
\hline$>45$ y & $18(2.6)$ & $4(2.3)$ & $7(3.7)$ & $7(2.1)$ \\
\hline \multicolumn{5}{|l|}{ Legal marital status } \\
\hline Never married & $592(84.7)$ & $141(81.0)$ & $169(88.5)$ & $282(84.4)$ \\
\hline Ever married/engaged & $107(15.3)$ & $33(19.0)$ & $2(11.5)$ & $52(15.6)$ \\
\hline \multicolumn{5}{|l|}{ Highest educational attainment } \\
\hline High school or below & $154(22.0)$ & 38 (21.8) & $44(23.0)$ & $72(21.6)$ \\
\hline Some college & $180(25.8)$ & 45 (25.9) & $51(26.7)$ & $84(25.1)$ \\
\hline Bachelor's degree and above & $365(52.2)$ & $91(52.3)$ & $96(50.3)$ & $178(53.3)$ \\
\hline \multicolumn{5}{|l|}{ Annual income (USD) ${ }^{a}$} \\
\hline$<\$ 5000$ ( low level ) & $208(29.8)$ & $47(27.0)$ & 55 (28.8) & $106(31.7)$ \\
\hline \$5001 9000 (lower-middle level) & $233(33.3)$ & $57(32.8)$ & $67(35.1)$ & $109(32.6)$ \\
\hline \$9001 14 000 (upper-middle level) & $153(21.9)$ & 34 (19.5) & $41(21.5)$ & $78(23.4)$ \\
\hline >\$14 000 (high level) & $105(15.0)$ & $36(20.7)$ & $28(14.7)$ & $41(12.3)$ \\
\hline \multicolumn{5}{|l|}{ Sexual orientation } \\
\hline Gay & $489(70.0)$ & $132(75.9)$ & $139(72.8)$ & $218(65.3)$ \\
\hline Bisexual & $178(25.4)$ & $35(20.1)$ & $43(22.5)$ & $100(29.9)$ \\
\hline Other & $32(4.6)$ & $7(4.0)$ & $9(4.7)$ & $16(4.8)$ \\
\hline Disclosure of sexual orientation to health provider & $307(43.9)$ & $80(46.0)$ & $93(48.7)$ & $134(40.1)$ \\
\hline Disclosure of sexual orientation to family or friends & $358(51.2)$ & $111(63.8)$ & $139(72.8)$ & $108(32.3)$ \\
\hline \multicolumn{5}{|l|}{ Number of male sex partners in the past 3 months } \\
\hline 0 & $117(16.7)$ & $21(12.1)$ & $33(17.3)$ & $63(18.9)$ \\
\hline 1 & $284(40.6)$ & $67(38.5)$ & $70(36.6)$ & $147(44.0)$ \\
\hline $2 \sim 5$ & 276 (39.5) & $80(46.0)$ & $78(40.8)$ & $118(35.3)$ \\
\hline$\geq 6$ & $22(3.2)$ & $6(3.4)$ & $10(5.2)$ & $6(1.8)$ \\
\hline Condomless anal intercourse in the past 3 months & $316(45.2)$ & 79 (45.4) & 85 (44.5) & $152(45.5)$ \\
\hline Female sex partner in the past 3 months & $109(15.6)$ & 26 (14.9) & $14(7.3)$ & $69(20.7)$ \\
\hline Ever used substances before or during sex & $355(50.8)$ & $96(55.2)$ & $98(51.3)$ & $161(48.2)$ \\
\hline Ever had group sex & $171(24.5)$ & $41(23.6)$ & $53(27.7)$ & $77(23.1)$ \\
\hline Ever had commercial sex (paid) & $130(18.6)$ & $40(23.0)$ & $24(12.6)$ & 66 (19.8) \\
\hline Ever had commercial sex (sold) & $112(16.0)$ & $36(20.7)$ & $14(7.3)$ & $62(18.6)$ \\
\hline Ever had HIV testing & $540(77.3)$ & $170(97.7)$ & $185(96.9)$ & $185(55.4)$ \\
\hline Ever had HIV self-testing & $406(58.1)$ & $168(96.6)$ & $101(52.9)$ & $137(41.0)$ \\
\hline Ever tested for syphilis in the hospital ${ }^{b}$ & $130(36.0)$ & $39(22.9)$ & 91 (47.6) & $\ldots$ \\
\hline Ever tested for syphilis in the community & $118(32.7)$ & 56 (32.9) & $62(32.5)$ & $\ldots$ \\
\hline
\end{tabular}

Data are presented as no. (\%)

Abbreviations: HIV, human immunodeficiency virus; USD, US dollar.

${ }^{a}$ The level of annual income (low, lower-middle, upper-middle, and high) was categorized by the standard from the National Bureau of Statistics (https://www.guancha.cn/ politics/2019_01_25_488206_1.shtml).

${ }^{\mathrm{b}}$ This analysis was restricted to participants who had ever tested for syphilis.

associated with syphilis self-testing are minimal. This study expands the literature by collecting data from MSM in many Chinese cities, focusing on syphilis self-testing, and exploring benefits and harms of using syphilis self-testing. Findings from this study provide insights for the implementation of syphilis self-testing programs and research among MSM in China.

We found that many Chinese MSM used syphilis selftesting. This rate of syphilis self-testing is higher than previously reported in the Netherlands [25] and several cities in China [18]. The high rate of syphilis self-testing may be related to widespread HIV self-testing [18], the high burden of syphilis in China [3], or the increasing availability of online syphilis self-testing kits [18]. Additionally, we found that men who had never received a facility-based syphilis test were more likely to use a syphilis self-test. This suggests that selftesting has the potential to reach populations that facilitybased strategies do not [28]. However, three-quarters of MSM in our sample had never used syphilis self-testing, suggesting that there is room for improvement on expanding coverage of syphilis self-testing services.

Our data suggest that nearly all of the syphilis self-testing was done alongside HIV self-testing. This is consistent with 
Table 2. Past Syphilis Self-test Experience, Posttest Health Services Utilization, and Potential Harms of Self-testing Among Chinese Men Who Have Sex With Men

\begin{tabular}{|c|c|c|}
\hline Attributes & No. & $\%$ \\
\hline \multicolumn{3}{|l|}{ Characteristics of Syphilis Self-testing } \\
\hline \multicolumn{3}{|l|}{ Location where syphilis self-test kit was obtained } \\
\hline Community-based organization & 117 & 67.2 \\
\hline Online drug store & 58 & 33.3 \\
\hline Hospital & 25 & 14.4 \\
\hline Friend & 11 & 6.3 \\
\hline Pharmacy & 6 & 3.5 \\
\hline \multicolumn{3}{|l|}{ Syphilis self-testing results (last self-test) } \\
\hline Reactive & 22 & 12.6 \\
\hline Not sure & 8 & 4.6 \\
\hline Negative & 144 & 82.8 \\
\hline \multicolumn{3}{|l|}{ Posttest actions } \\
\hline $\begin{array}{l}\text { Sought care following reactive/uncertain syphilis } \\
\text { self-testing result }(n=30)\end{array}$ & 25 & 83.3 \\
\hline \multicolumn{3}{|l|}{$\begin{array}{l}\text { Time since reactive/uncertain syphilis self-testing } \\
\text { result to seeking care }(n=25)\end{array}$} \\
\hline 0-2 weeks & 11 & 44.0 \\
\hline $2-4$ weeks & 7 & 28.0 \\
\hline $1-3$ months & 6 & 24.0 \\
\hline$>3$ months & 1 & 4.0 \\
\hline \multicolumn{3}{|l|}{ Location for seeking care $(n=25)$} \\
\hline General hospital & 9 & 36.0 \\
\hline Specialist STI service & 9 & 36.0 \\
\hline CDC & 3 & 12.0 \\
\hline Pharmacy/online counseling/others & 4 & 16.0 \\
\hline \multicolumn{3}{|l|}{ Benefits } \\
\hline Self-test as their first-time syphilis test & 90 & 51.7 \\
\hline Tested together with regular male sex partner & 92 & 52.9 \\
\hline Joined with HIV self-testing & 161 & 92.5 \\
\hline Increased syphilis testing uptake after first self-test & 49 & 28.2 \\
\hline \multicolumn{3}{|l|}{ Adverse events } \\
\hline Pressured testing & 30 & 17.2 \\
\hline \multicolumn{3}{|l|}{ Types of pressure } \\
\hline Physical violence & 6 & 3.4 \\
\hline Threats of violence & 7 & 4.0 \\
\hline Verbal abuse & 10 & 5.7 \\
\hline Psychological pressure & 6 & 3.4 \\
\hline Excessive control of activities & 5 & 2.9 \\
\hline Withholding of household resources & 3 & 1.7 \\
\hline Threatening to end a relationship & 10 & 5.7 \\
\hline \multicolumn{3}{|c|}{$\begin{array}{l}\mathrm{N}=174 . \\
\text { Abbreviations: CDC, Center for Disease Control and Prevention; HIV, human immunodefi- } \\
\text { ciency virus; STI, sexually transmitted infection. }\end{array}$} \\
\hline
\end{tabular}

previous studies $[18,25]$ suggesting that implementation of HIV self-testing programs could provide opportunities to encourage syphilis self-testing among MSM. However, among HIV self-testers in our study, only $40 \%$ of participants reported ever self-testing for syphilis. HIV self-testing has already created extensive infrastructure and public health pathways, including online testing programs, integration of public health and community-based organization programs, verification methods, and educational materials [29-31]. Given the syndemic of HIV and syphilis among MSM [32, 33], our data suggest that syphilis self-testing could be integrated into existing HIV self-testing platforms, organizations, and projects [9].

Currently, there are 3 main ways of disseminating self-testing kits, including through community-based organizations, e-commerce platforms, and pharmacies. In many LMICs [20], community-based organizations and public health programs are the main way of obtaining self-testing kits. This method is used in China [18], Zambia [34], Uganda [35], and Kenya [36]. E-commerce websites are another way of accessing self-test kits. This method is used in the United States (Amazon, Ebay) [37], China (Taobao, Jingdong) [37], and the Netherlands [25]. The third way is from pharmacies, which is done in the United States [38], the Netherlands [25], and the United Kingdom [39]. Although there are a number of ways of getting access to self-test kits, the lack of linkage to counseling, treatment, and care for self-test users who test positive is a concern for various stakeholders [40, 41].

Our study found minimal potential harms associated with syphilis self-testing. The frequency of pressured testing, violence, and coercion after testing observed in our sample was similar to that reported in HIV self-testing in Malawi [42] and in China [26-28]. Although the experience of being pressured to take STI tests was perceived to be negative, it could potentially lead to positive change and the adoption of risk-mitigation behaviors. A prior study documented that HIV testing frequency subsequently increased for the majority of participants who reported that they had been pressured to take an HIV test [27].

We used an online method to recruit participants for this study and our sample is more likely to be educated, younger, and have Internet access $[43,44]$. However, online technologies have been rapidly growing in use for sex-partner seeking among MSM [45]. Approximately $61.0 \%$ of MSM use online methods to seek sex partners in China $[46,47]$. Online methods are increasingly used because they can efficiently recruit large samples [43] and have some automation processes, which facilitate study implementation $[48,49]$. Our findings may not be generalizable to older MSM or other subgroups who are less often online. Nevertheless, we applied inverse probability of sampling weights and the G-formula to investigate the generalizability of results of a previous Chinese online MSM survey. We found that the results were similar when the previous online survey was quantitatively generalized to a national, cross-sectional survey dataset on MSM in China [50]. However, generalizations to other MSM populations should be done with caution. Future research may focus on this issue to provide more evidence for policy makers and practitioners [51].

Our study has several limitations. First, there might be selection bias in our study. Participants were recruited exclusively online. This sample might have been frequently exposed to HIV programs and studies, which had higher rates of HIV testing and self-testing compared with other surveys $[24,52,53]$. In 


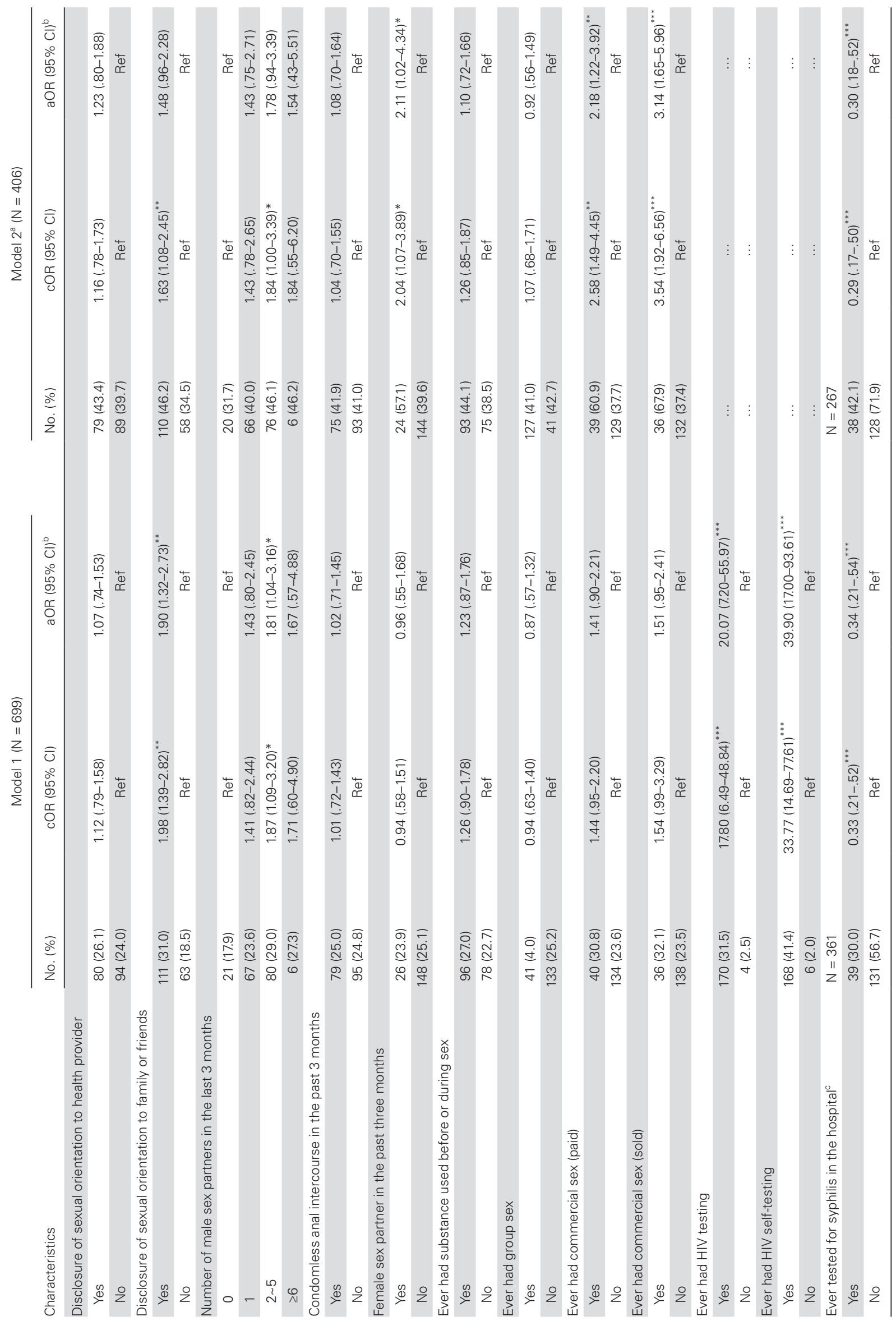




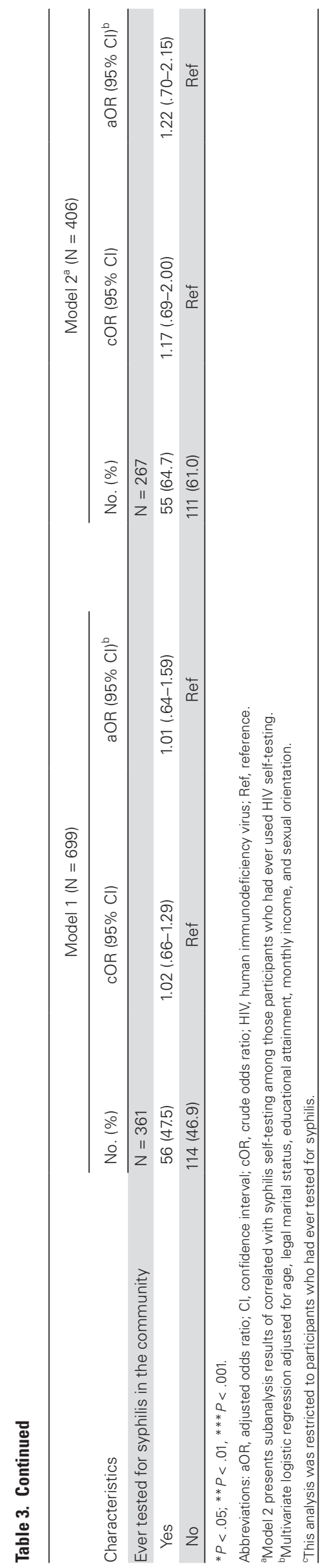

addition, we did not collect the information on the reasons that participants refused to attend the study. Those who refused to join the study may differ from the participants, which may have led to selection bias. Second, all of the data were obtained through self-report, which may be prone to information bias. However, in this study, we introduced a validation procedure for syphilis self-testing by verifying the images uploaded by testers. We found similar characteristics between those who had uploaded validated images and those who did not (Supplementary Table S4). Third, we have data from treponemal test results but not nontreponemal serologies, clinical data, or treatment history, which are important for differentiating new and old cases.

Our findings have implications for implementing syphilis self-testing among Chinese MSM. First, syphilis self-testing could be useful to help expand syphilis testing among MSM in China and in other countries that have an established HIV selftesting infrastructure. Second, self-testing might help facilitate couple-based testing services, which has been proven effective in reducing in HIV transmission risk, including among Chinese MSM [54, 55]. Third, incorporating syphilis self-testing into an HIV self-testing service has the potential to achieve the maximum benefits of the dual elimination of HIV and syphilis among MSM [56].

In conclusion, syphilis self-testing could be incorporated in China as a complement to facility-based testing services. Although the current syphilis testing rate is relatively low in MSM in China, this decentralized testing method could reach men and help expand syphilis testing. Syphilis self-testing can be integrated within HIV self-testing services to achieve further public health impact. Future studies to assess the effectiveness of syphilis self-testing in promoting syphilis prevention and control among MSM are warranted.

\section{Supplementary Data}

Supplementary materials are available at Clinical Infectious Diseases online. Consisting of data provided by the authors to benefit the reader, the posted materials are not copyedited and are the sole responsibility of the authors, so questions or comments should be addressed to the corresponding author.

\section{Notes}

Acknowledgments. The authors are grateful to all of the participants who participated in this study. The authors acknowledge Social Entrepreneurship for Sexual Health China, Shandong provincial Center for Disease Control and Prevention (CDC), Sichuan provincial CDC, Guizhou provincial CDC, Zhuhai CDC, Xinjiang Karamay City CDC, Xiamen Siming District CDC, Guangzhou Tongzhi, Lingnan Partner Community Support Center, Zhongda Sunshine group, Compass Organization, Suzhou Rainbow, Colorful Suzhou, Zhenjiang Rainbow, Changzhou Spring Breeze, Zhitong (Guangzhou and Xian), and Shenyang Love AID for helping with participant recruitment.

Financial support. This work was supported by the Dermatology Hospital of Southern Medical University and the National Institutes of Health (grant numbers NICHD UG3HD096929 and NIAID K24AI143471).

Potential conflicts of interest. The authors report no potential conflicts. All authors have submitted the ICMJE Form for Disclosure of Potential Conflicts of Interest. Conflicts that the editors consider relevant to the content of the manuscript have been disclosed.

2184 - CID 2020:70 (15 May) - Wang et al 


\section{References}

1. Peeling RW, Mabey D, Kamb ML, Chen XS, Radolf JD, Benzaken AS. Syphilis. Nat Rev Dis Primers 2017; 3:17073.

2. European Centre for Disease Prevention and Control (ECDC). Annual epidemiological report on communicable diseases in Europe. Stockholm: ECDC, 2018.

3. Chen G, Cao Y, Yao Y, et al. Syphilis incidence among men who have sex with men in China: results from a meta-analysis. Int J STD AIDS 2017; 28:170-8.

4. Jasek E, Chow EP, Ong JJ, et al. Sexually transmitted infections in Melbourne, Australia from 1918 to 2016: nearly a century of data. Commun Dis Intell Q Rep 2017; 41:E212-E22.

5. Center for Disease Control and Prevention. Sexually transmitted disease surveillance 2017 Atlanta: CDC, 2018.

6. Tucker JD, Hawkes SJ, Yin YP, Peeling RW, Cohen MS, Chen XS. Scaling up syphilis testing in China: implementation beyond the clinic. Bull World Health Organ 2010; 88:452-7.

7. World Health Organization. Guidelines on HIV self-testing and partner notification: supplement to consolidated guidelines on HIV testing services. Geneva: World Health Organization, 2016.

8. Zhang TP, Liu C, Han L, et al. Community engagement in sexual health and uptake of HIV testing and syphilis testing among MSM in China: a cross-sectional online survey. J Int AIDS Soc 2017; 20:21372.

9. Ong JJ, Fu H, Smith MK, Tucker JD. Expanding syphilis testing: a scoping review of syphilis testing interventions among key populations. Expert Rev Anti Infect Ther 2018; 16:423-32.

10. Song Y, Li X, Zhang L, et al. HIV-testing behavior among young migrant men who have sex with men (MSM) in Beijing, China. AIDS Care 2011;23:179-86.

11. Krause J, Subklew-Sehume F, Kenyon C, Colebunders R. Acceptability of HIV self-testing: a systematic literature review. BMC Public Health 2013; 13:735.

12. Myers JE, El-Sadr WM, Zerbe A, Branson BM. Rapid HIV self-testing: long in coming but opportunities beckon. AIDS 2013; 27:1687-95

13. Yang LG, Tucker JD, Wang C, et al. Syphilis test availability and uptake at medical facilities in southern China. Bull World Health Organ 2011; 89:798-805.

14. Newman L, Rowley J, Vander Hoorn S, et al. Global estimates of the prevalence and incidence of four curable sexually transmitted infections in 2012 based on systematic review and global reporting. PLoS One 2015; 10:e0143304.

15. Jamil MS, Prestage G, Fairley CK, et al. Effect of availability of HIV self-testing on HIV testing frequency in gay and bisexual men at high risk of infection (FORTH): a waiting-list randomised controlled trial. Lancet HIV 2017; 4:e241-50.

16. Tang W, Wu D. Opportunities and challenges for HIV self-testing in China. Lancet HIV 2018; 5:e611-2.

17. UNITAID, World Health Organization. Market and technology landscape: HIV rapid diagnostic tests for self-testing. 4th ed. Geneva: WHO/Unitaid, 2018

18. Zhong F, Tang W, Cheng W, et al. Acceptability and feasibility of a social entrepreneurship testing model to promote HIV self-testing and linkage to care among men who have sex with men. HIV Med 2017; 18:376-82.

19. Yin YP, Chen XS, Wei WH, et al. A dual point-of-care test shows good performance in simultaneously detecting nontreponemal and treponemal antibodies in patients with syphilis: a multisite evaluation study in China. Clin Infect Dis $\mathbf{2 0 1 3}$ 56:659-65

20. Tucker JD, Ong J, Conserve D, Pan S, Tang W. Scaling up HIV self-testing in China and Africa. J Virus Erad 2017; 3:167.

21. Tucker JD, Muessig KE, Cui R, et al. Organizational characteristics of HIV/syphilis testing services for men who have sex with men in South China: a social entrepreneurship analysis and implications for creating sustainable service models. BMC Infect Dis 2014; 14:601

22. Li KT, Tang W, Wu D, et al. Pay-it-forward strategy to enhance uptake of dual gonorrhea and chlamydia testing among men who have sex with men in China: a pragmatic, quasi-experimental study. Lancet Infect Dis 2019; 19:76-82.

23. Tucker JD, Wei C, Pendse R, Lo YR. HIV self-testing among key populations: an implementation science approach to evaluating self-testing. J Virus Erad 2015; $1: 38-42$.

24. Han L, Bien CH, Wei C, et al. HIV self-testing among online MSM in China: implications for expanding HIV testing among key populations. J Acquir Immune Defic Syndr 2014; 67:216-21.

25. Bil JP, Prins M, Stolte IG, et al. Usage of purchased self-tests for HIV and sexually transmitted infections in Amsterdam, the Netherlands: results of populationbased and serial cross-sectional studies among the general population and sexual risk groups. BMJ Open 2017; 7:e016609.

26. Ong JJ, Li H, Dan W, et al. Coercion and HIV self-testing in men who have sex with men: implementation data from a cross-sectional survey in China. J Acquir Immune Defic Syndr 2018; 77:e22-5.

27. Ong JJ, Wu D, Huang W, et al. Pressured HIV testing "in the name of love": a mixed methods analysis of pressured HIV testing among men who have sex with men in China. J Int AIDS Soc 2018; 21:e25098.
28. Qin Y, Tang W, Nowacki A, et al. Benefits and potential harms of human immunodeficiency virus self-testing among men who have sex with men in china: an implementation perspective. Sex Transm Dis 2017; 44:233-8.

29. Qin Y, Han L, Babbitt A, et al. Experiences using and organizing HIV self-testing. AIDS 2018; 32:371-81.

30. Johnson CC, Kennedy C, Fonner V, et al. Examining the effects of HIV selftesting compared to standard HIV testing services: a systematic review and metaanalysis. J Int AIDS Soc 2017; 20:21594.

31. Cheng WB, Xu HF, Zhong F, et al. [Application of "Internet Plus" AIDS preven tion services among men who have sex with men in Guangzhou, China: results from 2010 to 2015.] Zhonghua Yu Fang Yi Xue Za Zhi 2016; 50:853-7.

32. Chow EP, Tucker JD, Wong FY, et al. Disparities and risks of sexually transmissible infections among men who have sex with men in China: a meta-analysis and data synthesis. PLoS One 2014; 9:e89959.

33. Roberts CP, Klausner JD. Global challenges in human immunodeficiency virus and syphilis coinfection among men who have sex with men. Expert Rev Anti Infect Ther 2016; 14:1037-46.

34. Chanda MM, Ortblad KF, Mwale M, et al. HIV self-testing among female sex workers in Zambia: a cluster randomized controlled trial. PLoS Med 2017; 14:e1002442.

35. Ortblad K, Kibuuka Musoke D, Ngabirano T, et al. Direct provision versus facility collection of HIV self-tests among female sex workers in Uganda: a clusterrandomized controlled health systems trial. PLoS Med 2017; 14:e1002458.

36. Thirumurthy H, Masters SH, Mavedzenge SN, Maman S, Omanga E, Agot K. Promoting male partner HIV testing and safer sexual decision making through secondary distribution of self-tests by HIV-negative female sex workers and women receiving antenatal and post-partum care in Kenya: a cohort study. Lancet HIV 2016; 3:e266-74.

37. Liu F, Han L, Tang W, Huang S, Yang L, Zheng H. Availability and quality of online HIV self-test kits in China and the United States. In: Conference on Retroviruses and Opportunistic Infections. Seattle, US, 2015.

38. Myers JE, El-Sadr Davis OY, Weinstein ER, et al. Availability, accessibility, and price of rapid HIV self-tests, New York City pharmacies, summer 2013. AIDS Behav 2017; 21:515-24.

39. Ryan A, Wilson S, Greenfield S, Clifford S, McManus RJ, Pattison HM. Range of self-tests available to buy in the United Kingdom: an Internet survey. J Public Health 2006; 28:370-4.

40. UNAIDS. A short technical update on self-testing for HIV. Geneva: Joint United Nations Program on HIV. Technical brief. AIDS (London, England), 2013. https:// www.unaids.org/sites/default/files/media_asset/JC2603_self-testing_en_0.pdf

41. Napierala Mavedzenge S, Baggaley R, Corbett EL. A review of self-testing for HIV: research and policy priorities in a new era of HIV prevention. Clin Infect Dis 2013; 57:126-38.

42. Choko AT, MacPherson P, Webb EL, et al. Uptake, accuracy, safety, and linkage into care over two years of promoting annual self-testing for HIV in Blantyre, Malawi: a community-based prospective study. PLoS Med 2015; 12:e1001873.

43. Pequegnat W, Rosser BR, Bowen AM, et al. Conducting Internet-based HIV/STD prevention survey research: considerations in design and evaluation. AIDS Behav 2007; 11:505-21.

44. Wang C, Tucker JD, Liu C, Zheng H, Tang W, Ling L. Condom use social norms and self-efficacy with different kinds of male partners among Chinese men who have sex with men: results from an online survey. BMC Public Health 2018; 18:1175.

45. Guo Y, Li X, Stanton B. HIV-related behavioral studies of men who have sex with men in China: a systematic review and recommendations for future research. AIDS Behav 2011; 15:521-34.

46. Zhang W, Xu J-J, Zou H, Zhang J, Wang N, Shang H. HIV incidence and associated risk factors in men who have sex with men in Mainland China: an updated systematic review and meta-analysis. Sex Health 2016; 13:373-82.

47. Xu JJ, Tang WM, Zou HC, et al. High HIV incidence epidemic among men who have sex with men in china: results from a multi-site cross-sectional study. Infect Dis Poverty 2016; 5:82.

48. Shin E, Johnson TP, Rao K. Survey mode effects on data quality: comparison of web and mail modes in a US national panel survey. Soc Sci Comput Rev 2012; 30:212-28.

49. Bech M, Kristensen MB. Differential response rates in postal and Web-based surveys in older respondents. Survey Research Methods 2009; 3:1-6.

50. Wang C, Mollan KR, Hudgens MG, et al. Generalisability of an online randomised controlled trial: an empirical analysis. J Epidemiol Community Health 2018 $72: 173-8$.

51. Lesko CR, Buchanan AL, Westreich D, Edwards JK, Hudgens MG, Cole SR Generalizing study results: a potential outcomes perspective. Epidemiology 2017; 28:553-61.

52. Ren XL, Wu ZY, Mi GD, McGoogan J, Rou KM, Zhao Y. Uptake of HIV selftesting among men who have sex with men in Beijing, China: a cross-sectional study. Biomed Environ Sci 2017; 30:407-17. 
53. Yan H, Yang H, Raymond HF, et al. Experiences and correlates of HIV self-testing among men who have sex with men in Jiangsu province, China. AIDS Behav 2015; 19:485-91.

54. McMahon JM, Tortu S, Pouget ER, Torres L, Rodriguez W, Hamid R. Effectiveness of couple-based HIV counseling and testing for women substance users and their primary male partners: a randomized trial. Adv Prev Med 2013; 2013:286207.
55. Wei C, Muessig KE, Bien C, et al. Strategies for promoting HIV testing uptake: willingness to receive couple-based and collective HIV testing among a cross-sec tional online sample of men who have sex with men in China. Sex Transm Infect 2014; 90:469-74.

56. Taylor MM, Peeling RW, Toskin I, Ghinidelli M. Role of dual HIV/syphilis test kits in expanding syphilis screening. Sex Transm Infect 2017; 93:458-9. 